\title{
ESTRATÉGIA DE REDUÇÃO DO CUSTO DE TRANSPORTE DOS CENTROS DE DISTRIBUIÇÃO DA MARINHA DO BRASIL A PARTIR DE MÉTODOS HEURÍSTICOS
}

\author{
SANTOS, Marcos'1 ${ }^{1}$ RAMOS, Matheus Falcão²; REIS, Marcone Freitas ${ }^{3}$; WALKER, \\ Rubens Aguiar4 \\ ${ }^{1}$ Seção de Engenharia de Computação, Instituto Militar de Engenharia, marcosdossantos_doutorado_uff@yahoo.com.br \\ 2 Departamento de Engenharia de Produção, Faculdade SENAI CETIQT, mfalcaor@hotmail.com \\ 3 Departamento de Engenharia de Produção, Universidade Federal Fluminense, marconefreis11@gmail.com \\ ${ }^{4}$ Departamento de Engenharia Civil, Universidade Federal Fluminense, rubens.walker@gmail.com
}

Resumo: Embora a Marinha do Brasil seja uma instituição que não vise o lucro, seus gestores devem ter a preocupação de sempre melhorar os seus processos de maneira tratar com austeridade o erário público. Nesse sentido, sabendo que os custos de transporte representam mais da metade dos custos logísticos de uma organização, esse trabalho tem o objetivo de propor uma metodologia para redução dos custos de transporte, na região metropolitana do Rio de Janeiro, entre os centros de distribuição da Marinha do Brasil e as suas Organizações Militares (OM). O projeto piloto levou em consideração apenas o fornecimento de uniformes, na expectativa de ampliar o escopo para os demais itens de fornecimento. Realizou-se uma modelagem matemática preliminar na forma de um Problema de Programação Linear (PPL), e, ato contínuo, foram aplicados os métodos heurísticos do Canto Noroeste, do Menor Custo e da Aproximação de Voguel, na busca de uma Solução Básica Viável Inicial.

Palavras-chave: Pesquisa Operacional, Métodos Heurísticos, Custos de Transporte, Marinha do Brasil.

\section{STRATEGY TO REDUCE THE COST OF TRANSPORTATIONOF DISTRIBUTION CENTERS OF THE BRAZILIAN NAVY FROM HEURISTIC METHODS}

\begin{abstract}
Although the Brazilian Navy is an institution that does not aim for profit, its managers must be concerned to always improve their processes in a way that deals with the public treasury with austerity. In this sense, knowing that transport costs represent more than half of the logistics costs of an organization, this work has the objective of proposing a methodology to reduce transport costs, in the metropolitan region of Rio de Janeiro, between the distribution centers of the Navy of Brazil and its Military Organizations (MO). The pilot project took into account only the provision of uniforms, in the expectation of widening the scope for the other
\end{abstract}


items of supply. A preliminary mathematical modeling was developed in the form of a Linear Programming Problem (LPP), and the heuristic methods of the Northwest Corner, the Lower Cost and the Voguel Approximation were applied in the search for an Initial Basic Viable Solution.

Keywords: Operational Research, Heuristic Methods, Transport Costs, Brazilian Navy.

\section{Introdução}

O sistema de transporte se define como um conjunto de trabalho, facilidades e recursos que representam a capacidade de movimentação de cargas, pessoas e produtos intangíveis (energia, comunicação, telefonia, serviços) dentro da economia. (SILVA et al, 2016)

Segundo Hillier e Lieberman (2013), o problema de transporte (PT) é um modelo particularizado de um modelo de Programação Linear. O PT recebeu essa denominação em virtude de várias de suas aplicações envolverem problemas de transportar mercadorias de maneira otimizada, apesar do mesmo ter outras aplicações não relacionadas com transportes.

Para Simas (2013), o setor logístico no Brasil movimenta em torno de R 350 bilhões ao ano e este valor quase que duplicou nas últimas décadas, sendo responsável por transportar $60 \%$ de tudo o que é produzido no país .

A logística se destacou quando diversas empresas foram pressionadas pelo mercado a proporcionarem um atendimento diferenciado e um bom nível de atendimento. Nesse sentido, a sobrevivência das empresas passou a depender fortemente da busca contínua pela excelência no atendimento por meio de otimização de processos e formação de parcerias. (ASSUNÇÃO, ARANTES e SANTOS, 2016)

Alves e Zago (2016) relatam que o custo logístico do Brasil está em torno de 12,8\% do PIB, um valor bem acima de países como Estados Unidos com 8,2\% e Europa com 9\%.

A Programação Linear é uma ferramenta da Pesquisa Operacional (PO) que ajuda a solucionar os problemas de maximização e minimização de algum objetivo, atendendo a um conjunto de restrições. A modelagem é o método usado para a resolução do problema, elaborando um modelo matemático que melhor representa o desenvolvimento do mesmo. Propiciando assim resultados que poderão aprimorar o planejamento da produção e gerar soluções para tomadas de decisões. (LOESCH e HEIN, 2011)

Esta pesquisa tem por objetivo propor uma distribuição de suprimentos que minimize os custos de transporte entre os Centros de Distribuição e as Organizações Militares da Marinha do Brasil localizadas na cidade do Rio de Janeiro. 


\section{Problema}

A Marinha possui depósitos primários distribuídos pelo Rio de Janeiro. São esses depósitos que armazenam compras e, posteriormente, distribuem para todos as OM abastecidas por esses depósitos. Esses depósitos primários dividem-se em: Sobressalentes, Material Comum, Material de Subsistência, Material Eletrônico, Fardamento, Munição e Combustíveis. Estes dois últimos, Munição e Combustíveis, localizam-se em lugares isolados, em virtude dos cuidados especiais dos quais necessitam, pois são considerados materiais de risco e devem ser tratados com uma segurança elevada.

O seu complexo sistema de abastecimento, é gerenciado e desenvolvido pela Diretoria de Abastecimento da Marinha (DAbM), localizada no Primeiro Distrito Naval (Rio de Janeiro).

A recente crise econômica e fiscal pela qual o Brasil vem passando, evidencia a importância da austeridade no trato do erário público, de maneira a sempre utilizá-lo com a máxima eficiência e eficácia. Dado o exposto, surge o questionamento: Como minimizar os custos de transporte, dos Centro de Distribuição (CD) para as Organizações Militares?

$\mathrm{Na}$ busca da solução para esse problema, utilizou-se três métodos heurísticos, que possibilitam obter uma solução básica viável inicial para o problema em tela, indicando o volume de material que deve ser transportado de cada CD para cada OM de maneira a reduzir o custo do transporte. O mapa mental a seguir, Figura 1, mostra as condições de contorno do problema.

Figura 1 - Mapa Mental do Projeto

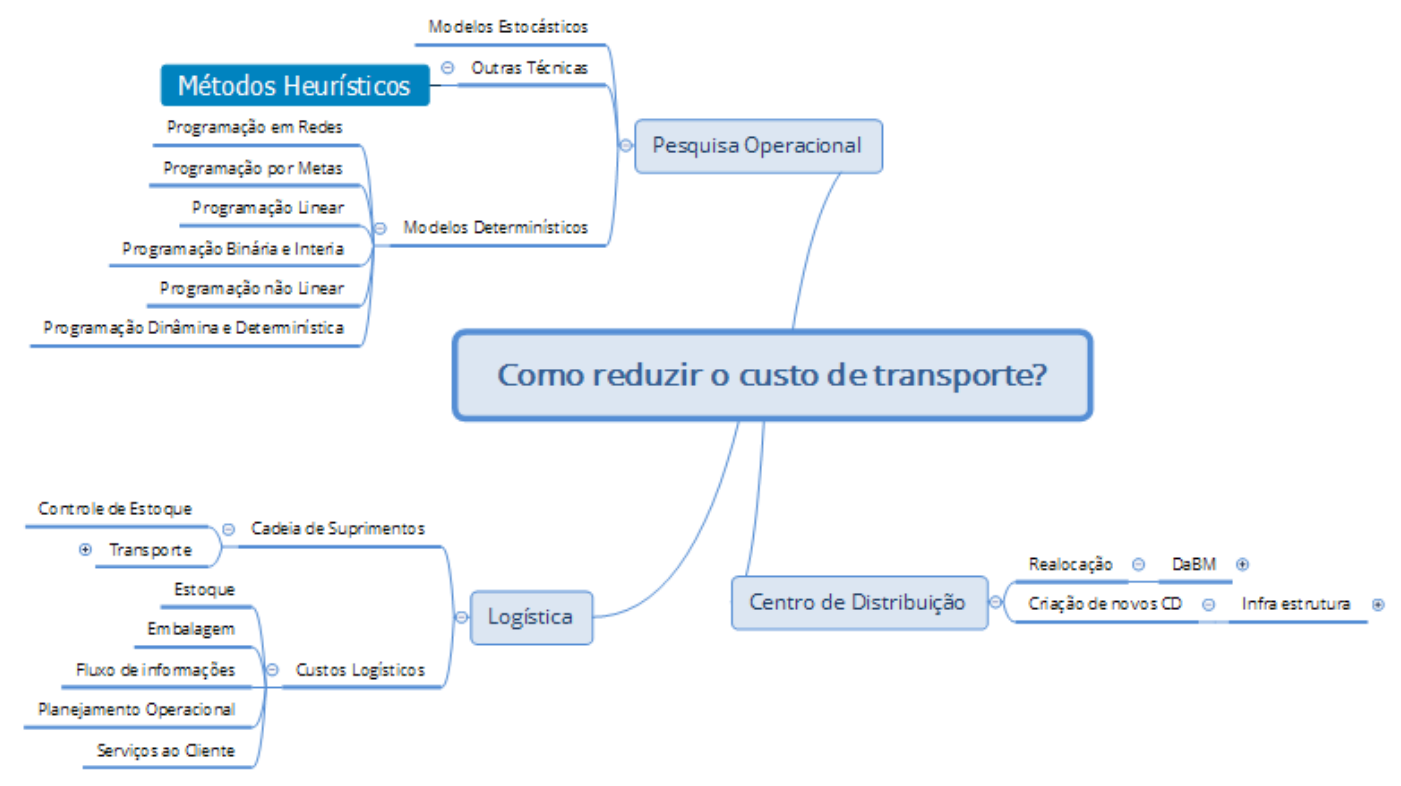

Fonte: Autores (2017) 


\section{Fundamentação Teórica}

Um engenheiro é, antes de mais nada, um "resolvedor de problemas". Ele tem a capacidade de compreender as condições de contorno de uma situação problemática e, a partir daí, propor soluções que agreguem valor não só para a organização da qual faz parte, mas também para a sociedade como um todo.

De acordo com Marins (2011) a Pesquisa Operacional, uma subárea da Engenharia de Produção, surgiu no Ocidente durante a Segunda Guerra Mundial (1939-1945), e é destinada às iniciativas dos serviços militares, tais como: desenvolvimento e uso de radar, problemas de dieta, problemas com alocação hábil de recursos escassos às diversas operações militares, entre outros.

Santos et al (2017) apresentam que todo problema de PO possui uma metodologia relativamente simples e logicamente encadeada, começando com uma situação problemática, ou seja, com um descompasso entre o que se quer e o que se tem, seja para uma pessoa, um grupo de pessoas ou uma organização. Assim, o primeiro passo na solução de um problema é o perfeito entendimento do mesmo e das suas condições de contorno.

Santos et al (2017) também afirmam que, fixado o objetivo, é necessário definir a medida de eficácia operacional (MEO), ou seja, uma medida que permita mensurar o quanto determinada medida contribui com o atingimento da função objetivo. Para cumprir essa função, a MEO deve possuir três características indispensáveis: deve ser quantificável, mensurável e completa. Quantificável diz respeito a ser possível representá-la por meio de um número. Mensurável, diz respeito a ser possível medila, de maneira direta ou indireta, pois de nada adianta uma MEO que pode ser quantificada, contudo não se tem como medi-la.

Para Loesch e Hein (2011), a utilização da PO é uma maneira de trazer conhecime nto para uma organização, uma vez que ela age como ferramenta de análise e estudo das problemáticas dos processos organizacionais. A utilização da $\mathrm{PO}$, como ciência, estrutura processos, propondo um conjunto de alternativas e ações, fazendo a previsão e a comparação de valores, de eficiência e de custos.

Segundo Lisboa (2012), um modelo é a representação simplificada de um sistema real, podendo ser um projeto já existente ou um projeto futuro. Contudo, modelagem visa a obtenção de subsídios para que o tomador de decisões identifique as limitações do mesmo e busca otimizar a eficiência do sistema. Programação linear, programação binária e inteira, metodologia multicritério de apoio a decisão, modelos de simulação e teoria das filas são exemplos de ferramentas utilizadas nos modelos de Pesquisa Operacional. 
Andrade (2009) afirma que a tomada de decisão é um processo sequencial e complexo, pois resulta de uma junção de várias decisões à cerca dos aspectos de dado problema e em uma inter-relação entre pessoas, responsabilidade pelo serviço, sistemas de informações, código de ética e moral, e comunicação, com valores subjetivos e desenvolvidos em um ambiente institucional com regras parcialmente definidas.

Segundo Biembengut e Hein (2003), a modelagem é um meio de interagir a matemática com a realidade, e tem a Figura 2 a seguir, como modo de representar esta proposta.

Figura 2 - Esquema do Processo de Modelagem Matemática

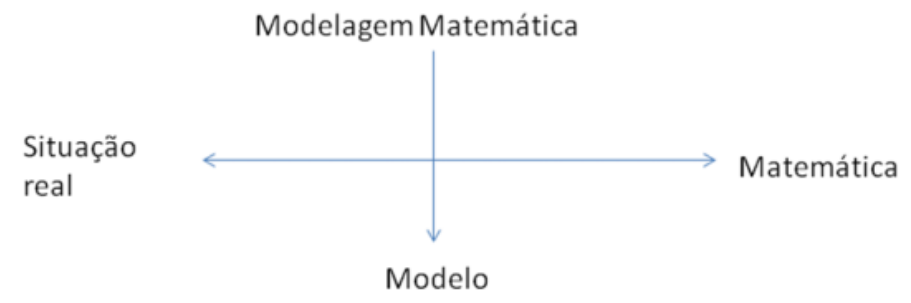

Fonte: Autores (2017)

O métodos heurísticos utilizados nesse trabalho justificam-se por se tratar de uma ferramenta de moderada complexidade matemática e que não exigem esforço computacional para a determinação de uma solução básica viável inicial.

\section{Modelagem Matemática}

\subsection{Dados do Modelo}

O desenvolvimento do trabalho teve como início uma análise dos dados fornecidos pela Marinha do Brasil. A partir desses dados, foi possível aplicar os métodos Heurísticos. A Tabela 1 apresenta cada Posto de Distribuição de Uniforme (PDU) e a respectiva capacidade de oferta de uniformes de cada um.

Tabela 1 - Dados da Capacidade de Oferta

\begin{tabular}{lc}
\hline \multicolumn{1}{c}{ Centros de Distribuição } & Capacidade de Oferta \\
\hline PDU BAMRJ & 36.000 \\
PDU Com1DN & 20.500 \\
PDU BNRJ & 27.500 \\
PDU Ilha do Governador & 13.000 \\
\hline Total & $\mathbf{9 7 . 0 0 0}$ \\
\hline
\end{tabular}

A Tabela 2 apresenta as demandas de cada $\mathrm{OM}$, situadas na região metropolitana do Rio de Janeiro, atendidas pelos quatro PDU. 
Tabela 2 - Dados da Capacidade de Demanda

\begin{tabular}{lc}
\hline \multicolumn{1}{c}{ Organizações Militares } & Capacidade de Demanda \\
\hline EGN & 710 \\
Escola Naval & 1.930 \\
CIAW & 3.200 \\
PNNSG & 840 \\
HNMD & 1.200 \\
Batalhão Toneleiro & 770 \\
Colégio Naval & 1.350 \\
Cluster Av. Brasil & 24.200 \\
Cluster Iha do Governador & 13.200 \\
Cluster Ponta da Areia & 4.600 \\
Cluster BNRJ & 26.000 \\
Cluster AMRJ & 19.000 \\
\hline Total & $\mathbf{9 7 . 0 0 0}$ \\
\hline
\end{tabular}

A Tabela 3 mostra o custo médio de transporte de cada peça de uniforme por quilômetro, além do custo de carregamento.

Tabela 3 - Dados Médios de Transporte e Carregamento por Peça

\begin{tabular}{lc}
\multicolumn{1}{c}{ Custos } & Preço \\
\hline Custo Médio de Transporte por km & $\mathrm{R} \$ 0,02$ \\
Custo Médio de Carregamento & $\mathrm{R} \$ 0,15$ \\
\hline
\end{tabular}

Fonte: Autores (2017)

\subsection{Variáveis de Decisão}

Como se deseja saber a quantidade a ser transportada de cada PDU para cada OM, as variáveis de decisão foram elencadas da seguinte maneira, conforme mostrado na Equação (1).

$X_{i j}=Q t$ tde de peças a serem transportadas da origem $i$ para o destino $j$ (1)

Em que:

$i:\{1,2,3,4\}$

$j:\{1,2,3,4,5,6,7,8,9,10,11,12\}$

\subsection{Restrições}

\section{Restrições de Oferta}

PDU da Base de Abastecimento da Marinha no Rio de Janeiro:

$$
\begin{aligned}
x_{1-1}+x_{1-2}+ & x_{1-3}+x_{1-4}+x_{1-5}+x_{1-6}+x_{1-7}+x_{1-8}+x_{1-9}+x_{1-10}+x_{1-11}+x_{1-12} \\
& \leq 36.000
\end{aligned}
$$

PDU do Comando do $1^{\circ}$ Distrito Naval: 


$$
\begin{aligned}
x_{2-1}+x_{2-2}+ & x_{2-3}+x_{2-4}+x_{2-5}+x_{2-6}+x_{2-7}+x_{2-8}+x_{2-9}+x_{2-10}+x_{2-11}+x_{2-12} \\
& \leq 20.500
\end{aligned}
$$

PDU da Base Naval do Rio de Janeiro:

$$
\begin{aligned}
x_{3-1}+x_{3-2}+ & x_{3-3}+x_{3-4}+x_{3-5}+x_{3-6}+x_{3-7}+x_{3-8}+x_{3-9}+x_{3-10}+x_{3-11}+x_{3-12} \\
& \leq 27.500
\end{aligned}
$$

PDU da Ilha do Governador:

$$
\begin{aligned}
x_{4-1}+x_{4-2}+ & x_{4-3}+x_{4-4}+x_{4-5}+x_{4-6}+x_{4-7}+x_{4-8}+x_{4-9}+x_{4-10}+x_{4-11}+x_{4-12} \\
& \leq 13.000
\end{aligned}
$$

\section{Restrições de Demanda}

Escola de Guerra Naval:

$$
x_{1-1}+x_{2-1}+x_{3-1}+x_{4-1} \leq 710
$$

Escola Naval:

$$
x_{1-2}+x_{2-2}+x_{3-2}+x_{4-2} \leq 1930
$$

Centro de Instrução Almirante Wandenkolk:

$$
x_{1-3}+x_{2-3}+x_{3-3}+x_{4-3} \leq 3200
$$

Policlínica Naval Nossa Senhora da Glória:

$$
x_{1-4}+x_{2-4}+x_{3-4}+x_{4-4} \leq 840
$$

Hospital Naval Marcílio Dias:

$$
x_{1-5}+x_{2-5}+x_{3-5}+x_{4-5} \leq 1200
$$

Batalhão Toneleiro:

$$
x_{1-6}+x_{2-6}+x_{3-6}+x_{4-6} \leq 770
$$

Colégio Naval:

$$
x_{1-7}+x_{2-7}+x_{3-7}+x_{4-7} \leq 1350
$$

Cluster Av. Brasil:

$$
x_{1-8}+x_{2-8}+x_{3-8}+x_{4-8} \leq 24200
$$

Cluster Ilha do Governador:

$$
x_{1-9}+x_{2-9}+x_{3-9}+x_{4-9} \leq 13200
$$

Cluster Ponta de Areia:

$$
x_{1-10}+x_{2-10}+x_{3-10}+x_{4-10} \leq 4600
$$

Cluster da Base Naval do Rio de Janeiro:

$$
x_{1-11}+x_{2-11}+x_{3-11}+x_{4-11} \leq 26000
$$

Cluster do Arsenal da Marinha no Rio de Janeiro:

$$
x_{1-12}+x_{2-12}+x_{3-12}+x_{4-12} \leq 19000
$$




\section{Restrições de Não Negatividade}

$$
\left\{x_{i j} \geq 0 ; \forall i ; \forall j\right\}
$$

\subsection{Função Objetivo}

Como se deseja minimizar o custo total de transporte, o qual é composto do custo de deslocamento mais o custo médio de carregamento por peça, chegou-se a seguinte função objetivo (F.O.):

$$
\begin{aligned}
& \text { F.O. }=\min \left\{0,55 x_{1-1}+0,492 x_{1-2}+0,426 x_{1-3}+0,402 x_{1-4}+0,452 x_{1-5}+0,954 x_{1-6}+\right. \\
& 3,01 x_{1-7}+0,15 x_{1-8}+0,442 x_{1-9}+0,616 x_{1-10}+0,662 x_{1-11}+0,44 x_{1-12}+0,354 x_{2-1}+ \\
& 0,262 x_{2-2}+0,176 x_{2-3}+0,294 x_{2-4}+0,416 x_{2-5}+1,132 x_{2-6}+3,29 x_{2-7}+ \\
& 0,422 x_{2-8}+0,586 x_{2-9}+0,544 x_{2-10}+0,588 x_{2-11}+0,15 x_{2-12}+0,6 x_{3-1}+ \\
& 0,542 x_{3-2}+0,478 x_{3-3}+0,48 x_{3-4}+0,602 x_{3-5}+1,222 x_{3-6}+3,39 x_{3-7}+0,542 x_{3-8}+ \\
& 0,708 x_{3-9}+0,706 x_{3-10}+0,15 x_{3-11}+0,492 x_{3-12}+0,804 x_{4-1}+0,68 x_{4-2}+ \\
& 0,63 x_{4-3}+0,598 x_{4-4}+0,658 x_{4-5}+1,262 x_{4-6}+3,57 x_{4-7}+0,43 x_{4-8}+0,174 x_{4-9}+ \\
& \left.0,794 x_{4-10}+0,838 x_{4-11}+0,616 x_{4-12}\right\}
\end{aligned}
$$

\subsection{Solução do Modelo}

Para obter uma solução básica viável inicial para o problema, foram utilizados três Métodos Heurísticos, são eles:

- Método do Canto Noroeste

- Método de Menor Custo

- Método de Aproximação de Vogel

\section{Método do Canto Noroeste}

A partir da Figura 3, aplicou-se o Método do Canto Noroeste. Na referida figura podese observar as capacidades dos PDU, as demandas das OM e o respectivo custo de transporte de cada PDU para cada OM.

Figura 3 - Aplicação do Método do Canto Noroeste

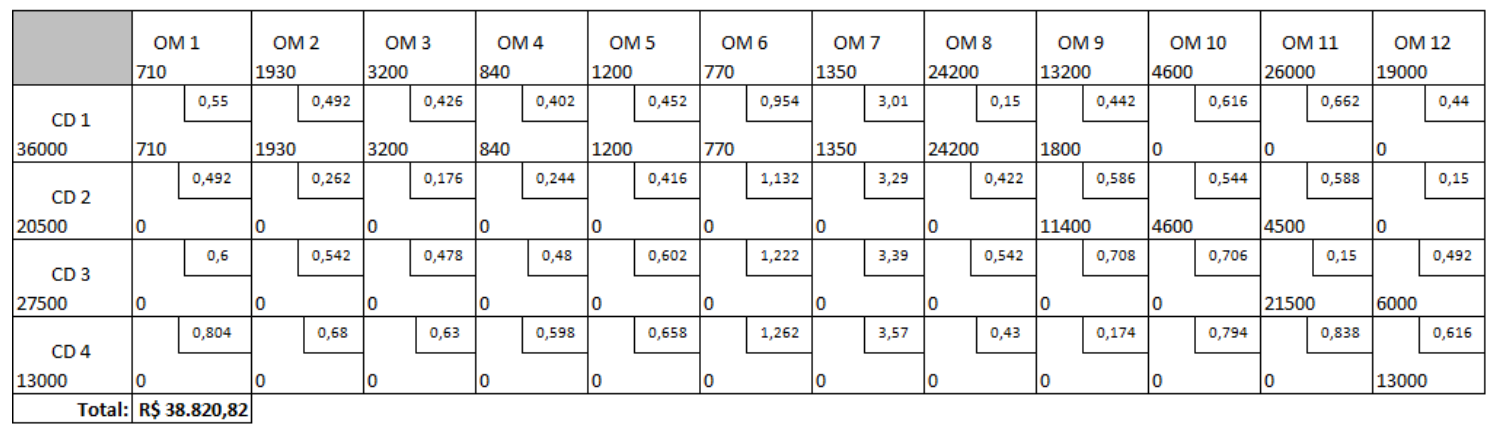

Fonte: Autores (2017) 
O Método do Canto Noroeste gerou a seguinte distribuição de transporte:

$$
\begin{gathered}
\mathrm{x}_{1-1}=710 \\
\mathrm{x}_{1-2}=1930 \\
\mathrm{x}_{1-3}=3200 \\
\mathrm{x}_{1-4}=840 \\
\mathrm{x}_{1-5}=1200 \\
\mathrm{x}_{1-6}=770 \\
\mathrm{x}_{1-7}=1350 \\
\mathrm{x}_{1-8}=24200 \\
\mathrm{x}_{1-9}=1800 \\
\mathrm{x}_{2-9}=11400 \\
\mathrm{x}_{2-10}=4600 \\
\mathrm{x}_{2-11}=4500 \\
\mathrm{x}_{3-11}=21500 \\
\mathrm{x}_{3-12}=6000 \\
\mathrm{x}_{4-12}=13000
\end{gathered}
$$

Tal distribuição gerou o seguinte custo de transporte:

Custo $=710 \times(0,55)+1930 \times(0,492)+3200 \times(0,426)+840 \times(0,402)+1200 \times(0,452)$ $+770 \times(0,954)+1350 \times(3,01)+24200 \times(0,15)+1800 \times(0,442)+11400 \times(0,586)+4600 \times$ $(0,544)+4500 \times(0,588)+21500 \times(0,15)+6000 \times(0,492)+13000 \times(0,616)=\mathbf{R} \$ \mathbf{3 8 . 8 2 0 , 8 2}$

\section{Método de Menor Custo}

A Figura 4 apresenta a aplicação do Método de Menor Custo no problema em estudo. Tal método é intuitivo por sempre alocar as maiores quantidades nos menores custos. No entanto, por ser um algoritmo guloso, normalmente conduz a resultados ruins.

Figura 4 - Aplicação do Método de Menor Custo

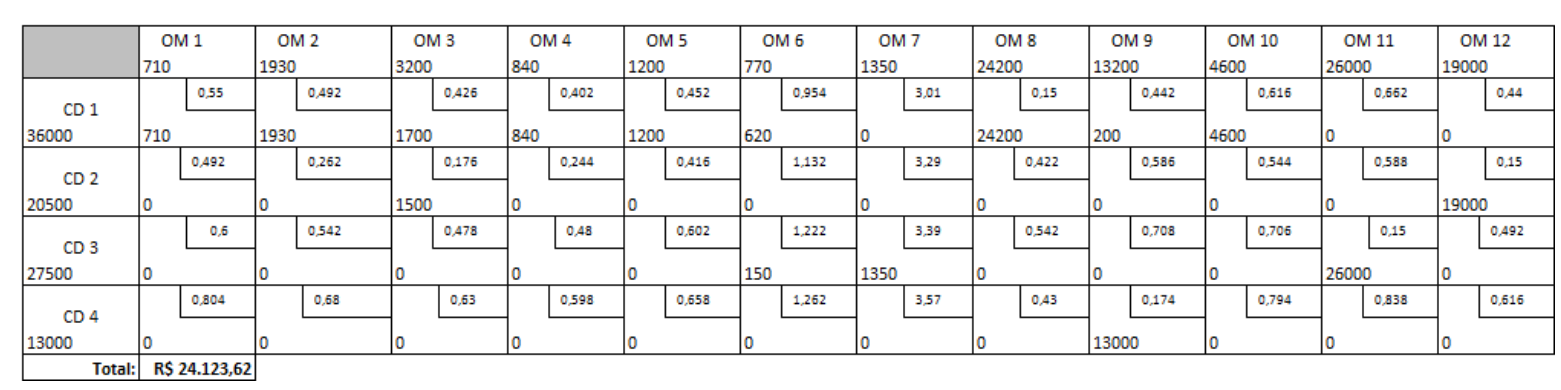

Fonte: Autores (2017)

Observa-se que tal método gera a seguinte distribuição de transporte: 


$$
\begin{gathered}
\mathbf{x}_{1-1}=\mathbf{7 1 0} \\
\mathbf{x}_{1-2}=1930 \\
\mathbf{x}_{1-3}=1700 \\
\mathbf{x}_{1-4}=\mathbf{8 4 0} \\
\mathbf{x}_{1-5}=1200 \\
\mathbf{x}_{1-6}=620 \\
\mathbf{x}_{1-8}=24200 \\
\mathbf{x}_{1-9}=\mathbf{2 0 0} \\
\mathbf{x}_{1-10}=4600 \\
\mathbf{x}_{2-3}=1500 \\
\mathbf{x}_{2-12}=19000 \\
\mathbf{x}_{3-6}=150 \\
\mathbf{x}_{3-7}=1350 \\
\mathbf{x}_{3-11}=26000 \\
\mathbf{x}_{4-9}=13000
\end{gathered}
$$

\begin{tabular}{|c|c|c|c|c|c|c|c|c|c|c|c|c|c|c|c|c|c|c|c|c|c|c|}
\hline & \multicolumn{2}{|c|}{$\begin{array}{l}\text { OM } 1 \\
710\end{array}$} & \multicolumn{2}{|c|}{$\begin{array}{c}\text { OM } 2 \\
1930\end{array}$} & \multicolumn{2}{|c|}{$\begin{array}{c}\text { OM } 3 \\
3200\end{array}$} & \multicolumn{2}{|c|}{$\begin{array}{l}\text { OM } 4 \\
840\end{array}$} & \multicolumn{2}{|c|}{$\begin{array}{c}\text { OM } 5 \\
1200\end{array}$} & \multicolumn{2}{|c|}{$\begin{array}{l}\text { OM } 6 \\
770\end{array}$} & \multicolumn{2}{|c|}{$\begin{array}{c}\text { OM 7 } \\
1350\end{array}$} & \multicolumn{2}{|c|}{$\begin{array}{c}\text { OM } 8 \\
24200\end{array}$} & $\begin{array}{c}\text { OM } 9 \\
13200\end{array}$ & \multicolumn{2}{|c|}{$\begin{array}{c}\text { OM } 10 \\
4600\end{array}$} & \multicolumn{2}{|l|}{$\begin{array}{c}\text { OM } 11 \\
26000\end{array}$} & $\begin{array}{c}\text { OM } 12 \\
19000\end{array}$ \\
\hline & & 0,55 & & 0,492 & & 0,426 & & 0,402 & & 0,452 & & 0,954 & & 3,01 & & 0,15 & 0,442 & & 0,616 & 0,66 & & 0,44 \\
\hline 36000 & 0 & & 114 & & & 700 & 84 & & & 200 & 77 & & 13 & & 242 & & 200 & 46 & & 0 & 0 & \\
\hline & & 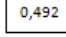 & & 0,262 & & 0,176 & & 0,244 & & 0,416 & & 1,132 & & 3,29 & & 0,422 & 0,586 & & 0,544 & 0,58 & & 0,15 \\
\hline 20500 & 0 & & 0 & & & 500 & 0 & & 0 & & 0 & & 0 & & 0 & & 0 & 0 & & 0 & & 9000 \\
\hline & & 0,6 & & 0,542 & & 0,478 & & 0,48 & & 0,602 & & 1,222 & & 3,39 & & 0,542 & 0,708 & & 0,706 & 0,13 & & 0,492 \\
\hline 27500 & 710 & & 796 & & 0 & & 0 & & 0 & & 0 & & 0 & & 0 & & 0 & 0 & & 26000 & 0 & \\
\hline & & \begin{tabular}{|l|l|} 
& 0,804 \\
\end{tabular} & & 0,68 & & 0,63 & & 0,598 & & 0,658 & & \begin{tabular}{|l}
, 262 \\
\end{tabular} & & 3,57 & & 0,43 & 0,174 & & 0,794 & 0,83 & & 0,616 \\
\hline 13000 & 0 & & 0 & & 10 & & 10 & & 10 & & 0 & & 0 & & 0 & & 13000 & 10 & & 0 & 10 & \\
\hline
\end{tabular}

Tal distribuição gera o seguinte custo de transporte:

Custo $=710 \times 0,55+1930 \times 0,492+1700 \times 0,426+1500 \times 0,176+840 \times 0,402+1200$ x $0,452+620 \times 0,954+150 \times 1,222+1350 \times 3,39+24200 \times 0,15+200 \times 0,442+13000 \times$ $0,174+4600 \times 0,616+26000 \times 0,15+19000 \times 0,15=\mathbf{R} \$ \mathbf{2 4 . 1 2 3 , 6 2}$

\section{Método de Aproximação de Vogel}

Aplicando o Método de Aproximação de Vogel foi obtida a distribuição inicial apresentada na Figura 5 a seguir.

Figura 5 - Aplicação do Método de Aproximação de Vogel

Fonte: Autores (2017)

Observa-se que tal método gera a seguinte distribuição de transporte: 


$$
\begin{gathered}
x_{1-2}=1140 \\
x_{1-3}=1700 \\
x_{1-4}=\mathbf{8 4 0} \\
x_{1-5}=\mathbf{1 2 0 0} \\
x_{1-6}=\mathbf{7 7 0} \\
x_{1-7}=1350 \\
x_{1-8}=\mathbf{2 4 2 0 0} \\
x_{1-9}=\mathbf{2 0 0} \\
x_{1-10}=4600 \\
x_{2-3}=1500 \\
x_{2-12}=19000 \\
x_{3-1}=\mathbf{7 1 0} \\
x_{3-2}=\mathbf{7 9 0} \\
x_{3-11}=\mathbf{2 6 0 0 0} \\
x_{4-9}=\mathbf{1 3 0 0 0}
\end{gathered}
$$

Tal distribuição gera o seguinte custo de transporte:

$$
\text { Custo }=710 \times(0,6)+1140 \times(0,492)+790 \times(0,542)+1700 \times(0,426)+1500 \times(0,176)
$$$$
+840 \times(0,402)+1200 \times(0,452)+770 \times(0,954)+1350 \times 3,01+24200 \times(0,15)+200 \times
$$$$
(0,442)+13000 \times(0,174)+4600 \times(0,616)+26000 \times(0,15)+19000 \times(0,15)=\mathbf{R} \$ \mathbf{2 3 . 6 4 5 , 4 2}
$$

\section{Discussão de Resultados}

Após a aplicação dos três métodos sugeridos no trabalho, pode-se verificar na Tabela 4 , a comparação dos resultados obtidos.

Tabela 4 - Resultado das Aplicações dos Métodos Propostos

\begin{tabular}{lll}
\hline \multicolumn{1}{c}{ Método } & Custo \\
\hline Método do Canto Noroeste & $\mathrm{R} \$ 38.820,82$ \\
Método de Menor Custo & $\mathrm{R} \$ 24.123,62$ \\
Método de Aproximação de Vogel & Fonte: Autores (2017) & \\
\hline
\end{tabular}

Tendo em vista os resultados apresentados, o melhor resultado obtido foi por meio do Método de Aproximação de Vogel, resultando em um custo total de transporte de R $23.645,42$. Tal resultado já era esperado, já que o Método de Aproximação de Voguel é mais sofisticado que os outros dois métodos, por trabalhar com o conceito de "penalidade".

\section{Considerações Finais}

No Brasil, os métodos analíticos da Pesquisa Operacional são conhecido há mais de cinquenta anos no âmbito acadêmico. Todavia, percebe-se uma certa resistência por parte da maioria das organizações, mesmo as de grande porte, como a Marinha do Brasil. 
O estudo ora apresentado, embora restrito apenas à distribuição de uniformes da Marinha do Brasil, mostrou que pode haver uma significativa economia dos recursos da Força, os quais poderiam ser investidos estrategicamente em outros setores. Tal economia mostra-se oportuna, dado o momento de forte contingenciamento pelo qual as Forças Armadas Brasileiras têm passado.

\section{Referências Bibliográficas}

ALVES, R.G., ZAGO, C.A. A Importância Da Roteirização No Nível De Serviço: Um Estudo Na Rga Operações Logísticas E Locação Ltda. Anais XXXVI Encontro Nacional De Engenharia De Produção. João Pessoa/PB, 2016.

ANDRADE, E. L. DE. Introdução à Pesquisa Operacional: Métodos e modelos para a análise de decisão. $4^{\mathrm{a}}$ Edição. Rio de Janeiro: Livros Técnicos e Científicos, 2009.

ASSUNCAO, M.A., ARANTES, B.R., SANTOS, M.F. Desenvolvimento De Ferramenta De Otimização Do Processo De Direcionamento De Frota Para Manutenção Anais XXXVI Encontro Nacional De Engenharia De Produção. João Pessoa/PB, 2016.

BIEMBENGUT, Maria Salett, HEIN, Nelson. Modelagem Matemática no ensino. 3.ed.São Paulo: Contexto, 2003.

HILLIER, F. S.; LIEBERMAN, G. J. Introdução à pesquisa operacional. Porto Alegre: AMGH, 2013.

LISBOA, Erico. Método Simplex. Rio de Janeiro: Universidade Estácio de Sá, 2012

LOESCH, C. e HEIN, N. Pesquisa Operacional: fundamentos e modelos. Blumenau: Editora Saraiva, 2011.

MARINS, Fernando Augusto Siva. Introdução à Pesquisa Operacional. São Paulo, 2011.

SANTOS, Marcos. Simulação da Operação de um Sistema Integrado de Informações para o atendimento pré-hospitalar de emergência no município do Rio de Janeiro. Dissertação de M.Sc., COPPE/UFRJ, Rio de Janeiro, Brasil, 2013.

SANTOS, Marcos dos; et al. O uso da Programação Linear Inteira (PLI) no apoio à Decisão e a Otimização do Mix de Produção. Anais do XXXVII Encontro Nacional de Engenharia de Produção - ENEGEP. Joinville/SC, 2017.

SILVA, E. M.; SILVA, E. M.; GONÇALVES, V.; MUROLO, A. C. Pesquisa Operacional: programação linear. 3 Ed. São Paulo: Atlas, 1998

SILVA, R.B., et al. Análise Multicritério Dos Motivadores Das Inovações No Sistema De Transporte No Setor De Logística. Anais XXXVI Encontro Nacional De Engenharia De Produção. João Pessoa/PB, 2016 